\title{
Data Grid Service
}

National Cancer Institute

\section{Source}

National Cancer Institute. Data Grid Service. NCI Thesaurus. Code C63544.

A grid service that provides an API with a query method, which allows the querying of any class or attribute in the exposed model. 